EXTENDED REPORT

\title{
Effects of brinzolamide on ocular haemodynamics in healthy volunteers
}

\author{
M Kaup, N Plange, M Niegel, A Remky, O Arend
}

Br J Ophthalmol 2004;88:257-262. doi: 10.1136/bjo.2003.021485

See end of article for authors' affiliations

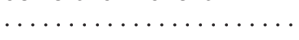

Correspondence to: Professor O Arend, Augenzentrum Alsdorf Cäcilienstrasse 9, 52477 Alsdorf, Germany; oliver.arend@web.de

Accepted for publication 3 July 2003

\begin{abstract}
Aim: A prospective, randomised study to evaluate effects of brinzolamide on ocular haemodynamics in healthy volunteers.

Methods: 30 volunteers (12 men, 18 women; 28.3 (SD 7.8) years) were prospectively randomised to either brinzolamide or placebo during a 2 week double masked treatment trial. Examinations were performed at baseline and after 2 weeks of treatment. Intraocular pressure was measured and automatic static perimetry (Humphrey field analyser, 24-2) and contrast sensitivity (CSV 1000, Vector Vision) were performed. Retrobulbar blood flow velocities (peak systolic and end diastolic velocity) and resistive indices (RI) of ophthalmic artery, central retinal artery and of temporal and nasal short posterior ciliary arteries were measured by colour Doppler imaging (Sonoline Sienna Siemens). In video fluorescein angiograms (scanning laser ophthalmoscope, Rodenstock) arteriovenous passage time (AVP, dilution curves) and peripapillary diameters of retinal arterioles and venules were measured by means of digital image analysis.

Results: Intraocular pressure was significantly decreased by brinzolamide $(p<0.0001)$. Neither brinzolamide nor placebo changed visual field global indices after treatment. Contrast sensitivity at 3 cycles per degree was significantly higher in the placebo group $(p<0.05)$. Apart from an increase of $R I$ in ophthalmic artery under placebo treatment $(p<0.05)$ there was no effect in retrobulbar haemodynamics in both groups. Brinzolamide therapy alone resulted in a significant reduction of AVP compared to baseline $(p<0.05)$, while peripapillary retinal vessels diameters remained unaffected.

Conclusions: Apart from the expected decrease of intraocular pressure brinzolamide showed no significant change in retrobulbar haemodynamics, but a significant shortening of AVP. Since in glaucoma AVP is prolonged indicating vascular dysfunction this effect might be beneficial in glaucoma therapy.
\end{abstract}

$\mathrm{T}$ he exact pathogenesis of glaucoma is still unknown. The development and progression of visual field loss and optic nerve damage are thought to be caused by several factors that include vascular abnormalities and dysregulation in addition to elevated intraocular pressure (IOP). ${ }^{1}$ Vascular deficits in retrobulbar vessels and in the ocular microcirculation have been observed in primary open angle glaucoma. ${ }^{2-7}$ For this reason, glaucoma medications should be evaluated for the hypotensive effect as well as for the effect on ocular haemodynamics.

Systemic carbonic anhydrase inhibitors (CAI), used in glaucoma therapy for almost 50 years, ${ }^{8}$ lower IOP by reducing the rate of aqueous humour secretion. In addition, they are known to have vasodilatory effects and to improve the blood flow in human retinal circulation. ${ }^{9}$ To reduce systemic side effects topical CAI, that lower IOP by inhibiting human carbonic anhydrase isoenzymes (CA) II and IV in the ciliary processes, were developed. ${ }^{10}$ Dorzolamide was the first available substance of this group. ${ }^{11}$ Several studies have presented effects of dorzolamide on ocular haemodynamics in humans. ${ }^{12-19}$ An acceleration of blood velocities in the retina, the superficial optic nerve head,,$^{12} 16$ and in retrobulbar vessels ${ }^{15}$ and an increase of ocular pulse amplitude ${ }^{13}$ were observed after topical application of dorzolamide. Contrasting results with no effect of dorzolamide on ocular blood flow presented in other studies ${ }^{17-19}$ might be the result of different techniques and study designs. Methods directed to different parts of ocular haemodynamics do not often allow direct comparisons of flow responses; thus, the effects of dorzolamide on ocular blood flow are still unclear.

Brinzolamide is a new topical CAI with a different physical profile than dorzolamide. ${ }^{20}$ It is more lipophilic and has low water solubility at $\mathrm{pH}$ 7.0-7.4. Therefore it is a suspension at physiological pH. Brinzolamide significantly reduced IOP in patients with primary open angle glaucoma and ocular hypertension. ${ }^{21}$ An animal study revealed that topical brinzolamide treatment resulted in an increase of the optic nerve head blood flow in rabbits. ${ }^{22}$ The effect of brinzolamide on ocular blood flow in humans is still unknown. Therefore we performed this prospective randomised study to evaluate the effects of brinzolamide on retinal and retrobulbar haemodynamics in healthy volunteers.

\section{PATIENTS AND METHODS}

Thirty healthy volunteers (12 men, 18 women; age 28 (SD 8) years) participated in this prospective randomised double masked study. All volunteers underwent a complete ophthalmological and general examination. The volunteers were instructed to avoid caffeine, tobacco, and exercise for at least 3 hours before the study visits. Informed consent was obtained from each volunteer including detailed explanation of all procedures before participation in this study. The protocol for the study was reviewed and approved by the institutional review board of University Aachen, Germany.

Abbreviations: AVP, arteriovenous passage time; $\mathrm{BP}$, blood pressure; CAl, carbonic anhydrase inhibitors; CDI, colour Doppler imaging; cpd, cycles per degree; CPSD, corrected pattern standard deviation; CRA, central retinal artery; $C S$, contrast sensitivity; EDV, end diastolic velocity; $\mathrm{HR}$, heart rate; MD, mean defect; OA, ophthalmic artery; OPP, ocular perfusion pressure; $P C A$, posterior ciliary artery; PSD, pattern standard deviation; PSV, peak systolic velocity; RI, resistive index; SF, short term fluctuation; SLO, scanning laser ophthalmoscope 
Table 1 Clinical data of blood pressure, heart rate, intraocular pressure, and calculated ocular perfusion pressure at baseline and after 2 weeks of treatment (mean (SD))

\begin{tabular}{|c|c|c|c|c|}
\hline & \multicolumn{2}{|c|}{ Brinzolamide } & \multicolumn{2}{|l|}{ Placebo } \\
\hline & Baseline & Drug & Baseline & Placebo \\
\hline $\begin{array}{l}\text { Systolic blood pressure } \\
(\mathrm{mm} \mathrm{Hg})\end{array}$ & $120(17)$ & $118(13)$ & $117(9)$ & $120(12)$ \\
\hline $\begin{array}{l}\text { Diastolic blood pressure } \\
\text { (mm Hg) }\end{array}$ & $73(11)$ & $71(8)$ & $70(7)$ & $72(9)$ \\
\hline Heart rate (beats $/ \mathrm{min}$ ) & $66(9)$ & $69(9)$ & $67(11)$ & $66(14)$ \\
\hline Intraocular pressure $(\mathrm{mm} \mathrm{Hg})$ & $12.3(2.0)$ & $9.4(2.2)^{*}$ & $13.2(1.6)$ & $12.3(2.2)$ \\
\hline $\begin{array}{l}\text { Ocular perfusion pressure } \\
(\mathrm{mm} \mathrm{Ha})\end{array}$ & $46.2(8.2)$ & $47.8(5.4)$ & $43.2(5.0)$ & $45.7(6.0)$ \\
\hline
\end{tabular}

Apart from a significant decrease of intraocular pressure after brinzolamide no significant differences between visits were detected in both groups (ANOVA for repeated measures). ${ }^{*} \mathrm{p}<0.0001$ (Bonferroni-Dunn).

The tenets of the Helsinki declaration were followed throughout the study.

During baseline examination visual acuity, contrast sensitivity (CS), automatic static perimetry (Humphrey field analyser), intraocular pressure (IOP), blood pressure, heart rate, colour Doppler imaging (CDI) and video fluorescein angiograms (scanning laser ophthalmoscope, SLO) were performed. The volunteers randomly received masked brinzolamide (Azopt, Alcon) or placebo (Lacrisic, Alcon) twice daily in the right eye. After 2 weeks of treatment all measurements were performed at the same time of day (plus or minus 30 minutes).

Best corrected visual acuity was tested using objective refractometry. Static contrast sensitivity (CSV 1000, Vector Vision, Dayton, OH, USA) was performed on all subjects at four spatial frequencies: 3, 6, 12, and 18 cycles per degree (cpd). ${ }^{23}$

The automatic static perimetry (program 24-2, conventional full threshold white on white) was performed with a Humphrey field analyser (Humphrey Inc, San Leandro, CA, USA). Global indices (mean defect (MD), pattern standard deviation (PSD), short term fluctuation (SF), and corrected pattern standard deviation (CPSD)) were statistically analysed.

Heart rate and blood pressure (BP) were determined by sphygmomanometry, with the patient in a sitting position after a rest of 5 minutes. Intraocular pressure (IOP) was measured before CDI and angiography examination using Goldmann applanation tonometry. Ocular perfusion pressure (OPP) was calculated from the blood pressure and intraocular pressure data $(\mathrm{OPP}=0.22$ (systolic $\mathrm{BP}+2 \times$ diastolic BP) - IOP).

Retrobulbar blood flow velocities of the ophthalmic artery (OA), central retinal artery (CRA) and temporal and nasal short posterior ciliary arteries (PCA) were measured by colour Doppler imaging (CDI). ${ }^{24}$ A $7.5 \mathrm{MHz}$ linear probe was applied to a closed eyelid using a coupling gel (Sonoline Sienna, Siemens). Samples of pulsed Doppler signal from within a $1.2 \times 1.2 \mathrm{~mm}$ sample volume were analysed to calculate blood velocities. In each vessel, peak systolic (PSV) and end diastolic velocity (EDV) were determined from the Doppler shifted spectral waveform. In addition, Pourcelot's resistive index (RI), a measure of peripheral vascular resistance, was calculated as follows: $\mathrm{RI}=(\mathrm{PSV}-\mathrm{EDV}) / \mathrm{PSV}$.

The OA was examined approximately $25 \mathrm{~mm}$ behind the globe at the straighter portion of the vessel in the nasal orbit to obtain the most reliable results. ${ }^{25}$ The CRA was measured behind the optic nerve head within the retrolaminar portion of the optic nerve. The short posterior ciliary arteries were detected 10-20 $\mathrm{mm}$ behind the globe nasal and temporal to the optic nerve where they commence as trunks before forming multiple branches surrounding the optic nerve in its retrobulbar portion. ${ }^{24}$

Digital scanning laser fluorescein angiography (Rodenstock Instruments, Ottobrunn, Germany) was performed to assess retinal arteriovenous passage time (AVP). The methodology was presented in detail elsewhere. ${ }^{26} 2.5 \mathrm{ml}$ of $10 \%$ fluorescein sodium dye was injected with a bolus of $5 \mathrm{ml}$ saline and the angiogram was videotaped. A series of frames was digitised from the videotape (Matrox Frame Grabber, Matrox Inc, Quebec, Canada). Mean intensity levels at the temporal superior and temporal inferior artery and corresponding veins over time were measured using a digital image analysis system (Adobe Photoshop, Adobe Systems Inc, San Jose, CA, USA). A rise in the plotted intensity curve corresponds to the appearance of fluorescein. The AVP characterises the fluorescein passage from the retinal artery through the capillary formation and arrival in the vein and is correlated with macular capillary blood velocity. ${ }^{27}$

The peripapillary diameters of the temporal superior and inferior vessels at the site of the measurement of the AVP were assessed from mid-transit fluorescein angiograms. A

Table 2 The conventional full threshold perimetry parameters (mean (SD) showed a significant difference of CPSD between brinzolamide and placebo after treatment (ANOVA for repeated measures), but neither brinzolamide nor placebo significantly changed CPSD in the subgroup analysis (Bonferroni-Dunn)

\begin{tabular}{llllll}
\hline & \multicolumn{2}{l}{ Brinzolamide } & & \multicolumn{2}{l}{ Placebo } \\
\cline { 2 - 3 } \cline { 5 - 6 } \cline { 5 - 6 } & Baseline & Drug & & Baseline & Placebo \\
\hline MD (dB) & $-1.00(0.85)$ & $-1.14(1.26)$ & & $-1.44(0.84)$ & $-0.88(0.81)$ \\
PSD (dB) & $1.68(0.27)$ & $1.79(0.58)$ & & $1.73(0.34)$ & $1.52(0.32)$ \\
SF (dB) & $1.47(0.58)$ & $1.20(0.36)$ & & $1.29(0.40)$ & $1.32(0.63)$ \\
CPSD (dB) & $0.79(0.62)$ & $1.19(0.72)$ & & $0.95(0.51)$ & $0.55(0.56)$ \\
\hline
\end{tabular}

$\mathrm{MD}=$ mean defect, $\mathrm{PSD}=$ pattern standard deviation, $\mathrm{SF}=$ short term fluctuation, $\mathrm{CPSD}=$ corrected pattern standard deviation. 
Table 3 Means of static contrast sensitivity at 3, 6, 12, and 18 cycles per degree (cpd) at baseline and after treatment

\begin{tabular}{llllll}
\hline & \multicolumn{3}{l}{ Brinzolamide } & & \multicolumn{2}{l}{ placebo } \\
\cline { 2 - 3 } \cline { 5 - 5 } & Baseline & Drug & & Baseline & Placebo \\
\hline $3 \mathrm{cpd}$ & $1.85(0.10)$ & $1.81(0.13)$ & & $1.78(0.17)$ & $1.85(0.13)^{*}$ \\
$6 \mathrm{cpd}$ & $1.93(0.47)$ & $2.04(0.18)$ & & $2.06(0.18)$ & $2.07(0.17)$ \\
$12 \mathrm{cpd}$ & $1.76(0.18)$ & $1.82(0.17)$ & & $1.75(0.33)$ & $1.85(0.21)$ \\
$18 \mathrm{cpd}$ & $1.34(0.20)$ & $1.35(0.12)$ & $1.33(0.22)$ & $1.34(0.18)$ \\
\hline Brinzolamide treatment showed no change in contrast sensitivity (ANOVA for repeated measures), in placebo \\
group contrast sensitivity at 3 cpd was significantly higher after treatment. \\
* $\mathrm{p}<0.05$ (Bonferroni-Dunn).
\end{tabular}

density profile was performed perpendicular to the vessel and by identifying the half height from the maximum height of the ascending and the descending slope diameter measurements. ${ }^{28}{ }^{29}$ To reach subpixel accuracy, the results represent the average of five separate diameter measurements. Results of AVP and vessel diameters from the temporal superior and inferior vessels were averaged to characterise posterior pole circulation.

Mean value and standard deviation were analysed for all samples using normal distributions (Kolmogorov-Smirnov test). For multiple comparisons ANOVA for repeated measures was used and $p$ values were obtained using BonferroniDunn test. Results of $\mathrm{p}<0.05$ were considered to be statistically significant.

\section{RESULTS}

Brinzolamide significantly reduced IOP after 2 weeks of treatment $(\mathrm{p}<0.0001)$, while the control group showed no change in IOP (table 1). Neither brinzolamide nor placebo changed visual field global indices, but there was a significant difference in CPSD between brinzolamide and placebo after treatment (table 2). Contrast sensitivity at $3 \mathrm{cpd}$ was significantly higher in placebo group after treatment $(p<0.05)$, while brinzolamide induced no changes at this frequency. No other frequency during CS testing showed any significance (table 3 ).

Brinzolamide and placebo left blood pressure, heart rate and calculated ocular perfusion pressure unaffected after 2 weeks of therapy. The clinical data are presented in table 1.
The retrobulbar haemodynamics measured by colour Doppler imaging showed no therapeutic effect by brinzolamide. Drug and placebo treatment left unaltered peak systolic and end diastolic velocities in ophthalmic artery, central retinal artery, and temporal and nasal posterior ciliary artery. In the placebo group an increase of RI in the ophthalmic artery was found after treatment $(\mathrm{p}<0.05)$, while brinzolamide left RI unchanged in any vessel (table 4).

The analysis of the video fluorescein angiograms at baseline and after treatment showed only after brinzolamide significant changes of retinal AVP. Brinzolamide therapy resulted in a significant reduction of AVP compared to baseline $(\mathrm{p}<0.05)$, while placebo had no significant effect on retinal passage time. In both groups peripapillary arterial and venous diameters remained unaffected during treatment. The results of the retinal parameters are presented in table 5 .

\section{DISCUSSION}

In this study we found that topical brinzolamide treatment in healthy volunteers lowers IOP and accelerates the retinal AVP, while the retinal vessel diameters and the retrobulbar haemodynamics remain unaltered.

Our results of shortened AVP during brinzolamide treatment agree with previous studies of the haemodynamic effects of dorzolamide. Decreased AVP coupled with unaltered retinal vessel diameter was associated with more rapid capillary transit in the macula and in superficial capillaries of the optic nerve head after dorzolamide treatment in normal subjects. ${ }^{12}$ These findings suggested that topical applied CAI

Table 4 Peak systolic velocity (PSV), end diastolic velocity (EDV) and resistive index (RI) in four retrobulbar vessels after double masked treatment with brinzolamide or placebo (mean (SD)

\begin{tabular}{|c|c|c|c|c|}
\hline & \multicolumn{2}{|l|}{ Brinzolamide } & \multicolumn{2}{|l|}{ Placebo } \\
\hline & Baseline & Drug & Baseline & Placebo \\
\hline \multicolumn{5}{|c|}{ Ophthalmic artery } \\
\hline $\operatorname{PSV}(\mathrm{cm} / \mathrm{s})$ & $29.8(5.9)$ & $28.4(6.2)$ & $32.1(7.0)$ & $31.6(8.0)$ \\
\hline $\operatorname{EDV}(\mathrm{cm} / \mathrm{s})$ & $5.8(1.9)$ & $5.6(1.4)$ & $6.0(2.3)$ & $5.1(1.2)$ \\
\hline RI & $0.81(0.06)$ & $0.80(0.05)$ & $0.81(0.07)$ & $0.83(0.05)^{*}$ \\
\hline \multicolumn{5}{|c|}{ Central retinal artery } \\
\hline $\operatorname{PSV}(\mathrm{cm} / \mathrm{s})$ & $9.6(2.1)$ & $10.0(2.1)$ & $9.8(1.2)$ & $10.3(1.4)$ \\
\hline $\operatorname{EDV}(\mathrm{cm} / \mathrm{s})$ & $2.9(1.2)$ & $3.2(0.9)$ & $2.9(0.6)$ & $2.9(0.7)$ \\
\hline $\mathrm{RI}$ & $0.70(0.09)$ & $0.68(0.07)$ & $0.70(0.06)$ & $0.72(0.08)$ \\
\hline \multicolumn{5}{|c|}{ Temporal posterior ciliary artery } \\
\hline $\operatorname{PSV}(\mathrm{cm} / \mathrm{s})$ & $9.1(2.1)$ & $9.5(1.8)$ & $8.3(2.0)$ & $8.4(2.1)$ \\
\hline $\operatorname{EDV}(\mathrm{cm} / \mathrm{s})$ & $3.6(1.3)$ & $3.7(1.2)$ & $3.0(0.8)$ & $2.9(0.7)$ \\
\hline $\mathrm{RI}$ & $0.60(0.07)$ & $0.62(0.08)$ & $0.63(0.07)$ & $0.64(0.08)$ \\
\hline \multicolumn{5}{|c|}{ Nasal posterior ciliary artery } \\
\hline $\operatorname{PSV}(\mathrm{cm} / \mathrm{s})$ & $8.2(2.5)$ & $8.1(2.1)$ & $7.6(1.1)$ & $7.8(1.2)$ \\
\hline EDV $(\mathrm{cm} / \mathrm{s})$ & $3.2(1.2)$ & $3.0(1.0)$ & $2.6(0.5)$ & $2.6(0.5)$ \\
\hline $\mathrm{RI}$ & $0.62(0.06)$ & $0.63(0.08)$ & $0.66(0.06)$ & $0.67(0.04)$ \\
\hline
\end{tabular}

Apart from an increase of RI in ophthalmic artery under placebo treatment there was no therapy effect in retrobulbar haemodynamics in both groups (ANOVA for repeated measures) * $p<0.05$ (Bonferroni-Dunn). 
Table 5 The results of the analysis of fluorescein angiograms at baseline and after treatment

\begin{tabular}{|c|c|c|c|c|}
\hline & \multicolumn{2}{|l|}{ Brinzolamide } & \multicolumn{2}{|l|}{ Placebo } \\
\hline & Baseline & Drug & Baseline & Placebo \\
\hline $\operatorname{AVP}(s)$ & $1.64(0.43)$ & $1.46(0.39)^{*}$ & $1.59(0.47)$ & $1.7(0.35)$ \\
\hline Retinal arterial diameter $(\mu \mathrm{m})$ & $103.2(13.8)$ & $102.6(12.7)$ & $106.1(13.4)$ & $101.9(10.4)$ \\
\hline Retinal vein diameter $(\mu \mathrm{m})$ & $139.3(15.2)$ & $138.3(16.3)$ & $136.2(13.8)$ & $136.9(13.0)$ \\
\hline
\end{tabular}

Brinzolamide therapy alone resulted in a significant shortening of AVP, while peripapillary retinal vessels diameters remained unaffected

${ }^{*} p<0.05$ (Bonferroni-Dunn).

enhances retinal perfusion. In patients with normal tension glaucoma dorzolamide treatment resulted in a shortening of AVP as well, ${ }^{14}$ while studies on the ocular haemodynamic effects of topical applied dorzolamide in open angle glaucoma patients presented contrasting results. ${ }^{16}{ }^{19}$ Furthermore, we demonstrated a reduction of AVP after a 4 week treatment of dorzolamide in patients with newly diagnosed primary open angle glaucoma. ${ }^{16}$ Bergstrand et al found no measurable vascular effects from a 6 week treatment of dorzolamide in previously untreated glaucoma eyes. ${ }^{19}$ This difference of circulatory results after dorzolamide treatment in patients with open angle glaucoma may be accounted for variable study designs, ethnic populations, and algorithms for analysis of the collected data. Prospective studies are necessary to evaluate effects of brinzolamide treatment on ocular blood flow in patients with glaucoma.

The retrobulbar haemodynamics measured by colour Doppler imaging showed no therapeutic effect of brinzolamide. These findings imply that the brinzolamide induced acceleration of retinal AVP is not promoted via changes in the retrobulbar blood flow velocities. On the other hand the intraindividual variability of the methodology may be too high to detect small changes in retrobulbar arteries. ${ }^{25}$ The changes presented in the retinal circulation were not related to significant differences in the peripheral resistance of these vessels. Identical results were previously presented for dorzolamide in healthy volunteers ${ }^{12}$ and patients with glaucoma. ${ }^{14^{19}}{ }^{30}$ In contrast, Galassi et al found a significant decrease of RI in the temporal posterior ciliary artery after a 4 week treatment with dorzolamide in patients with newly diagnosed primary open angle glaucoma. ${ }^{31}$ The authors suggested that the resistance decrease might be due to a vasodilatory effect of dorzolamide in the choroidal vascular bed or to the reduction of IOP. An increase in retrobulbar blood flow velocities after topical application of dorzolamide was shown in an unmasked study in normal and glaucomatous subjects. ${ }^{15}$ The possibility remains open that retrobulbar haemodynamics, shown to be abnormal in several studies of primary open angle glaucoma, ${ }^{36}$ could be normalised by CAI. In healthy volunteers even systemic CAI failed to modify blood flow velocities or RI in retrobulbar vessels ${ }^{32}$; thereby, it is not surprising that topical applied brinzolamide left the retrobulbar haemodynamics unaltered.

The current study showed no change of contrast sensitivity after brinzolamide treatment, but an improvement at $3 \mathrm{cpd}$ after placebo as it was reported for artificial tear film at this spatial frequency in normal eyes. ${ }^{33}$ Dorzolamide appeared to enhance contrast sensitivity in normal subjects under physiological hypercapnia and hypocapnia. ${ }^{34}$ In patients with glaucoma dorzolamide improves the contrast sensitivity. ${ }^{14} 16$ It is unknown if IOP reduction or altered ocular perfusion might directly or indirectly cause the improvement in central visual function. No correlation was found between these effects of dorzolamide. ${ }^{14}$
Brinzolamide treatment resulted in a shortening of AVP. This reduction associated with unchanged retinal arterial and venous diameters suggests that the drug enhances retinal perfusion. Even AVP itself is only an indirect marker of total retinal perfusion. The analysis of AVP, however, allows us to detect haemodynamic alterations closely related to clinical abnormalities in glaucoma. Patients with open angle glaucoma showed prolonged retinal passage time compared with healthy subjects. ${ }^{27}$ These circulatory deficits of the retinal tissue are linked together with visual field loss. In hemispheres with more severe glaucomatous field loss the AVP was significantly prolonged compared with the less affected hemisphere. ${ }^{7}$ Since prolonged AVP has been associated with disease progression in glaucoma ${ }^{75}{ }^{36}$ the visual function may benefit from increased retinal perfusion by brinzolamide seen in shortened AVP. Studies of long term application of brinzolamide are needed to evaluate the effects on ocular blood flow and visual function in glaucoma patients.

To date, a comparative study of different topical applied CAI on ocular blood flow has been presented in rabbits, but not in humans. ${ }^{22}$ The IOP reduction, as well as the improvement of optic nerve head blood flow in rabbits, was statistically equivalent after treatment with either brinzolamide or dorzolamide. ${ }^{22}$ Both CAI were found in the retina after topical administration to rabbits, but in different concentrations, ${ }^{11} 20$ though further studies are needed to confirm these results because of methodological differences. In humans, no differences were found in the IOP effects of the two CAI, whereas brinzolamide appeared to be slightly more effective in reducing aqueous humour flow than dorzolamide. ${ }^{37}$ A comparative study between brinzolamide and dorzolamide is necessary to detect possible different effects on ocular blood flow in humans.

Brinzolamide lowers IOP by locally inhibiting the CA II and CA IV in ciliary processes and suppressing aqueous humour secretion. ${ }^{20}$ The mechanism by which brinzolamide may increase retinal perfusion is unclear. Ocular isoenzymes that could be inhibited would be membrane bound CA IV in the retinal pigment epithelium ${ }^{38}$ and vascular endothelium of the choriocapillaris ${ }^{39}$ and the soluble CA II in the Mueller cells of the retina. ${ }^{40}$ The inhibition of CA II in the Mueller cells by topical applied CAI is uncertain because the diffusion across the blood-retinal barrier would depend on the lipophilicity and an adequate concentration of free drug. Since brinzolamide is more lipophilic than dorzolamide at physiological $\mathrm{pH}^{20}$ this higher lipophilicity may favour the ability to move across lipid membrane barriers. ${ }^{41}$ Brinzolamide has its highest affinity and inhibitory potency for CA II. The potency for inhibiting CA IV is comparable to dorzolamide. ${ }^{20}$ It is unclear, if different properties of CA inhibition may influence the effects on ocular blood flow.

Systemic CAI induced a significant increase in retinal blood flow measured by laser Doppler velocimetry in healthy volunteers combined with a vasodilatation of retinal vessels 
determined from monochromatic fundus photographs. ${ }^{9}$ Rassam et al concluded that the mechanisms responsible for the increase in retinal blood flow acted via significant increases in perfusion pressure, red cell velocity, and retinal vessel dilatation. An increase in tissue $\mathrm{pCO}_{2}$ and a reduction in $\mathrm{pH}$ were thought to be responsible for the vascular dilatation as it was postulated for the effects of acetazolamide on cerebral circulation. ${ }^{42}$ The animal study in tranquillised Dutch belted rabbits revealed that brinzolamide and dorzolamide significantly increased optic nerve head blood flow measured by fundus camera based laser Doppler flowmeter. ${ }^{22}$ The authors suggested that the enhanced optic nerve head blood flow might be the result of a possible increase in ocular tissue carbon dioxide tension. The carbon dioxide and $\mathrm{pH}$ mediated mechanism may account for the shortening of AVP in humans by brinzolamide. In a study of hypercapnia in healthy volunteers AVP fell and mean arterial dye velocity and capillary blood velocity rose as $\mathrm{pCO}_{2}$ increased. ${ }^{43}$ Anderson and Davis found that an increase of local $\mathrm{pCO}_{2}$ in cell cultured bovine pericyte monolayer caused a relaxation of the pericytes in a reversible, concentration dependent manner. ${ }^{44}$ Therefore, part of local blood flow regulation may reside in the capillary bed through the contractile properties of pericytes. Recently, Reber et al showed that CAI caused an increase of retinal capillary diameter coupled with a decrease of extracellular and an increase of intracellular $\mathrm{pH}$ in rat retinal organ culture. ${ }^{45}$ They suggested that CAI could relax pericytes and might improve the retinal blood supply. Based on these findings the brinzolamide induced acceleration of AVP with unaltered peripapillary retinal vessel diameters may be due to a vasodilatory effect in the retinal capillary bed.

In conclusion, a 2 week treatment with brinzolamide in healthy volunteers resulted in the expected reduction of IOP and in a shortening of retinal AVP, while the peripapillary retinal vessel diameters and the retrobulbar haemodynamics remained unaltered. Since in glaucoma AVP is prolonged indicating vascular dysfunction brinzolamide treatment may benefit optic nerve head preservation by increasing retinal perfusion seen in shortened retinal AVP. Further testing of brinzolamide treatment on ocular blood flow and visual function in patients with glaucoma is needed.

\section{ACKNOWLEDGEMENT}

This study was supported financially in part by Alcon Pharma GmbH, Freiburg iB, Germany.

\section{Authors' affiliations}

M Kaup, N Plange, M Niegel, A Remky, O Arend, Department of Ophthalmology, Aachen University, Aachen, Germany

\section{REFERENCES}

1 Flammer J, Orgül S. Optic nerve blood-flow abnormalities in glaucoma. Prog Retin Eye Res 1998; 17:267-89.

2 Wolf $\mathrm{S}$, Arend $\mathrm{O}$, Sponsel WE, et al. Retinal hemodynamics using scanning laser ophthalmoscopy and hemorheology in chronic open-angle glaucoma. Ophthalmology 1993;100:1561-6.

3 Harris A, Sergott RC, Spaeth GL, et al. Color Doppler analysis of ocular vessel blood velocity in normal-tension glaucoma. Am J Ophthalmol 1994; 118:642-9.

4 Kaiser HJ, Schoetzau A, Stumpfig D, et al. Blood-flow velocities of the extraocular vessels in patients with high-tension and normal-tension primary open-angle glaucoma. Am J Ophthalmol 1997;123:320-7.

5 Grunwald JE, Piltz J, Hariprasad SM, et al. Optic nerve and choroidal circulation in glaucoma. Invest Ophthalmol Vis Sci 1998;39:2329-36.

6 Rankin SJ. Color Doppler imaging of the retrobulbar circulation in glaucoma. Surv Ophthalmol 1999;43(Suppl 1):S176-82.

7 Arend O, Remky A, Cantor LB, et al. Altitudinal visual field asymmetry is coupled with altered retinal circulation in patients with normal pressure glaucoma. Br J Ophthalmol 2000;84:1008-12.

8 Becker B. Decrease in intraocular pressure in man by carbonic anhydrase inhibitor, Diamox. Am J Ophthalmol 1954;37:13-15.
9 Rassam SM, Patel V, Kohner EM. The effect of acetazolamide on the retinal circulation. Eye 1993;7(P+ 5):697-702.

10 Maren TH. The development of topical carbonic anhydrase inhibitors. J Glaucoma 1995;4:49-62.

11 Sugrue MF. The preclinical pharmacology of dorzolamide hydrochloride, a topical carbonic anhydrase inhibitor. J Ocul Pharmacol Ther 1996; 12:363-76.

12 Harris A, Arend O, Arend S, et al. Effects of topical dorzolamide on retinal and retrobulbar hemodynamics. Acta Ophthalmol Scand 1996;74:569-72.

13 Schmidt KG, von Ruckmann A, Pillunat LE. Topical carbonic anhydrase inhibition increases ocular pulse amplitude in high tension primary open angle glaucoma. Br J Ophthalmol 1998;82:758-62.

14 Harris A, Arend O, Kagemann L, et al. Dorzolamide, visual function and ocular hemodynamics in normal-tension glaucoma. $J$ Ocul Pharmacol Ther 1999; 15:189-97.

15 Martinez A, Gonzalez F, Capeans C, et al. Dorzolamide effect on ocular blood flow. Invest Ophthalmol Vis Sci 1999;40:1270-5.

16 Arend O, Harris A, Wolter P, et al. Evaluation of retinal haemodynamics and retinal function after application of dorzolamide, timolol, and latanoprost in newly diagnosed open angle glaucoma patients. Acta Ophthalmol Scand 2003; (in press).

17 Grunwald JE, Mathur S, DuPont J. Effects of dorzolamide hydrochloride 2\% on the retinal circulation. Acta Ophthalmol Scand 1997;75:236-8.

18 Pillunat LE, Bohm AG, Koller AU, et al. Effect of topical dorzolamide on optic nerve head blood flow. Graefes Arch Clin Exp Ophthalmol 1999;237:495-500.

19 Bergstrand IC, Heiil A, Harris A. Dorzolamide and ocular blood flow in previously untreated glaucoma patients: a controlled double-masked study. Acta Ophthalmol Scand 2002;80:176-82.

20 DeSantis L. Preclinical overview of brinzolamide. Surv Ophthalmol 2000:44(Suppl 2):S119-29.

21 March WF, Ochsner KI. The long-term safety and efficacy of brinzolamide $1.0 \%$ (Azopt) in patients with primary open-angle glaucoma or ocular hypertension. The Brinzolamide Long-Term Therapy Study Group. Am J Ophthalmol 2000;129:136-43.

22 Barnes GE, Li B, Dean T, et al. Increased optic nerve head blood flow after 1 week of twice daily topical brinzolamide treatment in Dutch-belted rabbits. Surv Ophthalmol 2000;44(Suppl 2):S131-40

23 Pomerance GN, Evans DW. Test-retest reliability of the CSV-1000 contrast test and its relationship to glaucoma therapy. Invest Ophthalmol Vis Sci 1994;35:3357-61.

24 Williamson TH, Harris A. Color Doppler ultrasound imaging of the eye and orbit. Surv Ophthalmol 1996;40:255-67.

25 Harris A, Williamson TH, Ophth FC, et al. Test/retest reproducibility of color Doppler imaging assessment of blood flow velocity in orbital vessels. J Glaucoma 1995:4:281-6.

26 Wolf $\mathrm{S}$, Jung $\mathrm{F}$, Kiesewetter $\mathrm{H}$, et al. Video fluorescein angiography: method and clinical application. Graefes Arch Clin Exp Ophthalmol 1989;227:145-51

27 Arend O, Harris A, Martin BJ, et al. Scanning laser ophthalmoscopy-based evaluation of epipapillary velocities: method and physiologic variability. Surv Ophthalmol 1999;44(Suppl 1):S3-9.

28 Delori FC, Fitch KA, Feke GT, et al. Evaluation of micrometric and microdensitometric methods for measuring the width of retinal vessel images on fundus photographs. Graefes Arch Clin Exp Ophthalmol 1988;226:393-9.

29 Arend O, Remky A, Plange N, et al. Capillary density and retinal diameter measurements and their impact on altered retinal circulation in glaucoma: a digital fluorescein angiographic study. $\mathrm{Br} J$ Ophthalmol 2002;86:429-33.

30 Harris A, Arend O, Chung HS, et al. A comparative study of betaxolol and dorzolamide effect on ocular circulation in normal-tension glaucoma patients. Ophthalmology 2000;107:430-4.

31 Galassi F, Sodi A, Renieri G, et al. Effects of timolol and dorzolamide on retrobulbar hemodynamics in patients with newly diagnosed primary openangle glaucoma. Ophthalmologica 2002;216:123-8.

32 Harris A, Tippke S, Sievers $\mathrm{C}$, et al. Acetazolamide and $\mathrm{CO}_{2}$ : acute effects on cerebral and retrobulbar hemodynamics. J Glaucoma 1996;5:39-45.

33 Huang FC, Tseng SH, Shih MH, et al. Effect of artificial tears on corneal surface regularity, contrast sensitivity, and glare disability in dry eyes. Ophthalmology 2002; 109:1934-40.

34 Sponsel WE, Harrison J, Elliott WR, et al. Dorzolamide hydrochloride and visual function in normal eyes. Am J Ophthalmol 1997;123:759-66.

35 Arend O, Remky A, Redbrake C, et al. [Retinal hemodynamics in patients with normal pressure glaucoma. Quantification with digital laser scanning fluorescein angiography]. Ophthalmologe 1999;96:24-9.

36 Arend O, Remky A, Harris A. Retinal hemodynamics in normal tension glaucoma. In: Pillunat LE, Harris A, Anderson DR, Greve EL, eds. Current concepts on ocular blood flow in glaucoma. The Hague: Kugler Publications, 1999:245-9

37 Ingram CJ, Brubaker RF. Effect of brinzolamide and dorzolamide on aqueous humor flow in human eyes. Am J Ophthalmol 1999;128:292-6.

38 Wolfensberger TJ, Mahieu I, Jarvis-Evans J, et al. Membrane-bound carbonic anhydrase in human retinal pigment epithelium. Invest Ophthalmol Vis Sci 1994;35:3401-7.

39 Hageman GS, Zhu XL, Waheed A, et al. Localization of carbonic anhydrase IV in a specific capillary bed of the human eye. Proc Natl Acad Sci USA 1991;88:2716-20. 
40 Lutien-Decroll E, Lonnerholm G. Carbonic anhydrase distribution in the rabbit eye by light and electron microscopy. Invest Ophthalmol Vis Sci 1981;21:782-97.

41 Eller MG, Schoenwald RD, Dixson JA, et al. Topical carbonic anhydrase inhibitors. III: Optimization model for corneal penetration of ethoxzolamide analogues, J Pharm Sci 1985;74:155-60.

42 Vorstrup S, Henriksen L, Paulson OB. Effect of acetazolamide on cerebral blood flow and cerebral metabolic rate for oxygen. J Clin Invest 1984;74:1634-9.
43 Harris $\mathrm{A}$, Arend $\mathrm{O}$, Wolf $\mathrm{S}$, et al $\mathrm{CO}_{2}$ dependence of retinal arterial and capillary blood velocity. Acta Ophthalmol Scand 1995;73:421-4.

44 Anderson DR, Davis EB. Glaucoma, capillaries and pericytes. 5. Preliminary evidence that carbon dioxide relaxes pericyte contractile tone. Ophthalmologica 1996;210:280-4.

45 Reber F, Gersch U, Funk RW. Blockers of carbonic anhydrase can cause increase of retinal capillary diameter, decrease of extracellular and increase of intracellular $\mathrm{pH}$ in rat retinal organ culture. Graefes Arch Clin Exp Ophthalmol 2003;241:140-8.

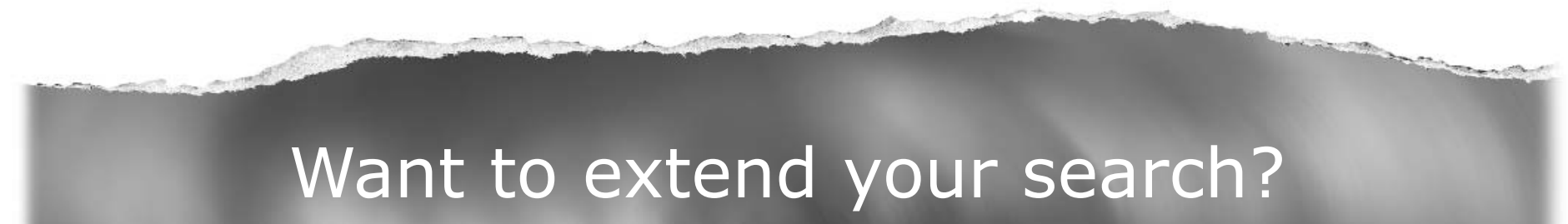

\section{Cross journal searching}

Can't find what you're looking for in British Journal of Ophthalmology? Extend your search across 340+ journals. Search restriction options include specific subject areas (eg. clinical medicine, basic research), select specific journals or search all available titles.

www.bjophthalmol.com 